\title{
February 2016 Critical Care Case of the Month
}

\section{Thomas M. Stewart, MD \\ Bhargavi Gali, MD}

\author{
Department of Anesthesiology \\ Mayo Clinic Minnesota \\ Rochester, MN USA
}

\section{Case Presentation}

A 32 year-old, previously healthy, female hospital visitor had been participating in a family care conference regarding her critically ill grandmother admitted to the cardiac intensive care unit. During the care conference, she felt unwell and had some mild chest discomfort; she collapsed and cardiopulmonary resuscitation (CPR) was initiated (1). Upon arrival of the code team, she was attached to the monitor and mask ventilation was initiated. Her initial rhythm is shown in Figure 1.

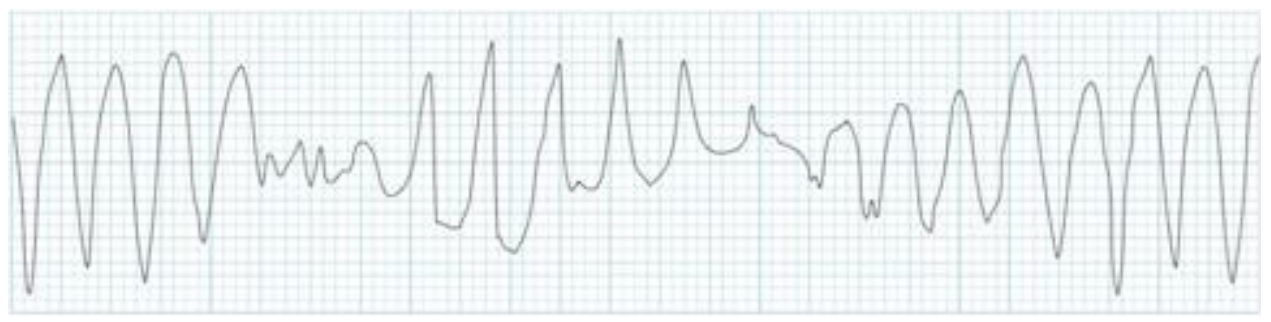

Figure 1. Initial rhythm strip.

In addition to DC cardioversion which of the following should be administered immediately?

1. Lidocaine

2. Magnesium sulfate

3. Procainamide

4. 1 and 3

5. All of the above 


\section{Correct! \\ 2. Magnesium sulfate}

The rhythm is torsades de pointes, or torsades, an uncommon and distinctive form of polymorphic ventricular tachycardia (VT) characterized by a gradual change in the amplitude and twisting of the QRS complexes around the isoelectric line (2). Torsades is associated with a prolonged QT interval, usually terminates spontaneously but frequently recurs and may degenerate into ventricular fibrillation.

The etiology and management of torsades are different from those of usual ventricular tachycardia. Magnesium is the drug of choice in torsades. Magnesium achieves this by decreasing the influx of calcium. Group IA antiarrythmics such as procainamide and quinidine tend to prolong the QT interval, and can worsen torsades. Differentiating between these entities, therefore, is critically important. Lidocaine is usually without effect in torsades.

Intravenous access was established and magnesium $4 \mathrm{gm}$ was administered. She was defibrillated a total of 3 times. Throughout the resuscitative efforts she had respiratory effort, and she was assisted with bag valve mask. Return of spontaneous circulation was achieved after 10-12 minutes of CPR.

Which of the following are associated with torsades?

1. Azithromycin

2. Jervell and Lange-Nielsen syndrome

3. Romano Ward syndrome

4. 1 and 3

5. All of the above 


\section{Correct! \\ 5. All of the above}

The Jervell and Lange-Nielsen syndrome and the Romano Ward syndrome are congenital abnormalities associated with QT prolongation and sudden death. The former is associated with congenital deafness while the later is not. A number of drugs have been associated with QT prolongation and torsades, most recently macrolide antibiotics (3).

Our patient was conscious post-arrest and her vital signs were stable. 12-Lead electrocardiogram revealed slight ST-elevation in the anteroseptal leads that resolved within an hour but no QT prolongation. Transthoracic echocardiogram showed no obvious wall motion abnormality. Cardiac MRI and cardiac CT angiography were negative for abnormalities. Eventually, she underwent cardiac catheterization and was found to have spontaneous coronary artery dissection (SCAD) in the left main coronary artery, the mid left anterior descending coronary artery, and involvement of the left circumflex coronary artery as well (Figure 2 ).

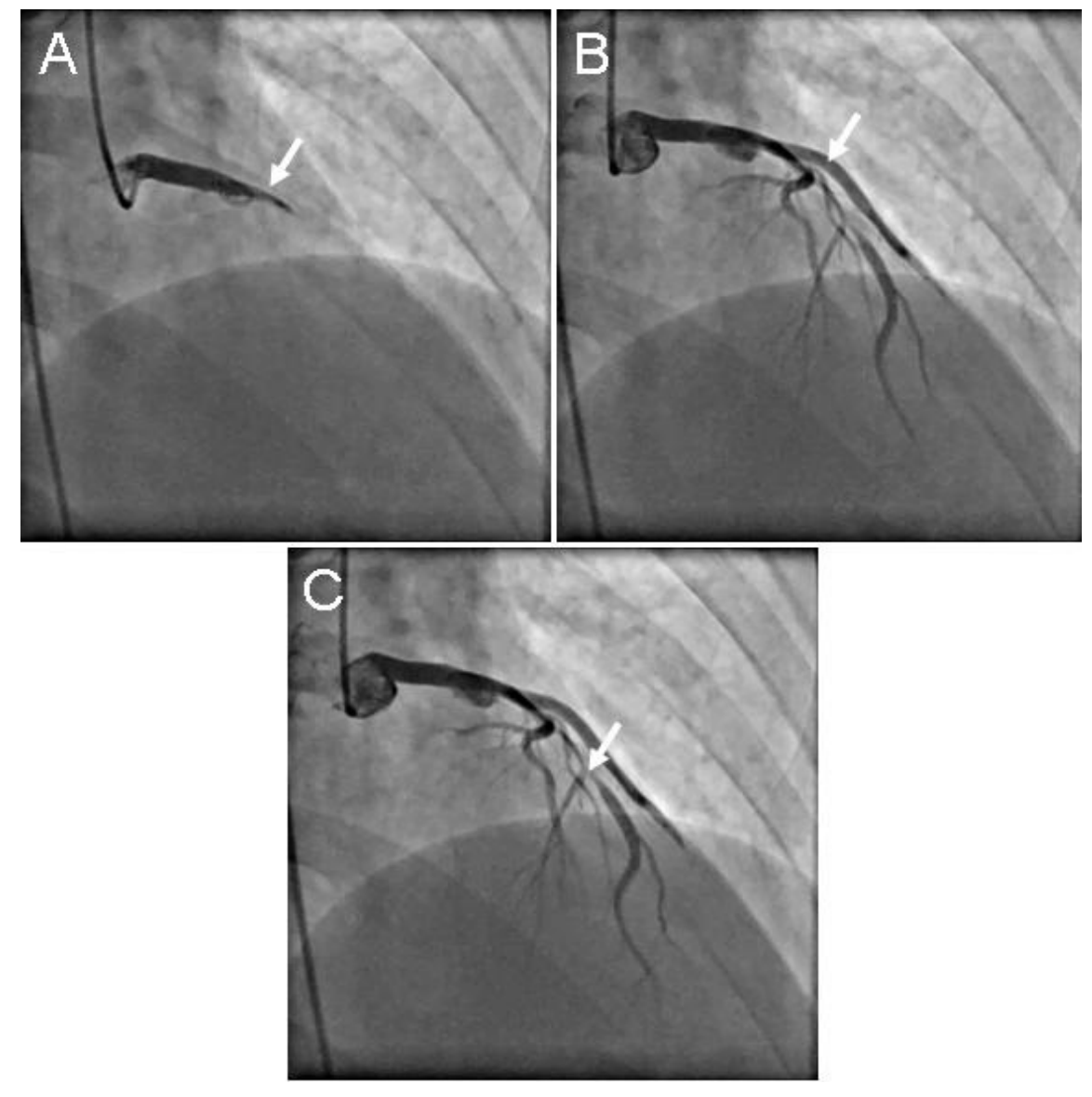

Figure 2. Static views of the coronary angiogram showing narrowing from the coronary artery dissection of the left main (Panel A), anterior descending and cirumflex coronary arteries. 
Which of the following have been associated with spontaneous coronary artery dissection?
1. Cocaine
2. Ehlers-Danlos syndrome
3. Fibromuscular dysplasia
4. 1 and 3
5. All of the above 


\section{Correct! \\ 5. All of the above}

Spontaneous coronary artery dissection is a rare cause of acute coronary syndrome with a spectrum of clinical presentations ranging from chest pain to ST-elevation myocardial infarction to ventricular fibrillation and sudden death (4). It typically affects a younger, otherwise healthy, female population. Initial mortality is fairly low, however tenyear rates of major adverse cardiac events is estimated at $47 \%$. Fibromuscular dysplasia, connective tissue disorders such as Ehlers-Danlos or Marfan syndrome, and cocaine use have all been associated and are potentially causative factors.

Surgical versus medical management was discussed. Percutaneous intervention carried too great a risk for further progression of the dissection. Coronary artery bypass graft surgery carried a high risk of early graft failure due to competitive flow. Given these potential complications, medical management was pursued.

\section{References}

1. Hazinski MF, Nolan JP, Aickin R, et al. Part 1: Executive summary: 2015 international consensus on cardiopulmonary resuscitation and emergency cardiovascular care science with treatment recommendations. Circulation. 2015;132(16 Suppl 1):S2-39. [CrossRef] [PubMed]

2. Dave J, Bessette M, Setnik G, Gaeta TJ, Lakhia R. Torsades des pointes. Medscape (2015). Available at: http://emedicine.medscape.com/article/1950863overview (accessed 1/26/16).

3. Yap YG, Camm AJ Drug induced QT prolongation and torsades de pointes. Heart. 2003;89(11):1363-72. [CrossRef] [PubMed]

4. Tweet MS, Hayes SN, Pitta SR, et al. Clinical features, management, and prognosis of spontaneous coronary artery dissection. Circulation. 2012;126(5):579-88. [CrossRef] [PubMed] 